MPP-2004-15

\title{
Double Suppression of FCNCs in Supersymmetric Models
}

\author{
Ki-Young Choi ${ }^{1,2}$, Yuji Kajiyama ${ }^{3}$, Jisuke Kubo ${ }^{3,4}$, and Hyun Min Lee ${ }^{2}$ \\ ${ }^{1}$ School of Physics and Center for Theoretical Physics, \\ Seoul National University, Seoul 151-747, Korea \\ ${ }^{2}$ Physikalisches Institut, Universität Bonn, D-53115 Bonn, Germany \\ ${ }^{3}$ Institute for Theoretical Physics, Kanazawa University, Kanazawa 920-1192, Japan \\ 4 Max-Planck-Institut für Physik, Werner-Heisenberg-Institut, D-80805 Munich, Germany
}

\begin{abstract}
A mechanism for double suppression of flavor-changing neutral currents (FCNCs) and CP violating phases in supersymmetric models is suggested. At $M_{\mathrm{SUSY}}$ they are suppressed due to a nonabelian discrete flavor symmetry, and the infrared attractive force of gauge interactions in extra dimensions are used to suppress them at the compactification scale. We present a concrete model, which is a simple extension of $S_{3}$ invariant minimal supersymmetric standard model, where only $S U(2)_{L}$ and $S U(3)_{C}$ gauge multiplets are assumed to propagate in the bulk. We find that a disorder of two orders of magnitude in soft supersymmetry breaking parameters above the compactification scale may be allowed to satisfy experimental constraints on FCNC processes and CP violating phenomena.
\end{abstract}

PACS numbers: $12.60 . \mathrm{Jv}, 11.25 . \mathrm{Mj}, 11.30 . \mathrm{Hv}$ 


\section{INTRODUCTION}

Low energy supersymmetry (SUSY) is introduced to protect the Higgs mass from the quadratic divergence [1]. Since low energy SUSY is broken, the breaking of SUSY must be soft, whatever its origin is, to maintain the very nature of low energy SUSY. Unfortunately, the most arbitrary part of a phenomenologically viable supersymmetric extension of the standard model (SM) is this soft breaking sector, because renormalizability allows an introduction of many soft supersymmetry breaking (SSB) parameters. In the minimal supersymmetric standard model (MSSM), more than 100 SSB parameters can be introduced

2]. The problem is not only this large number of the SSB parameters, but also the fact that one has to highly fine tune them so that they do not induce unacceptably large flavor changing neutral currents (FCNCs) and $\mathrm{CP}$ violations [3, 4, 5, 6, 7]. This problem, called the SUSY flavor problem, is not new, but has existed ever since supersymmetry found phenomenological applications [8].

There are several theoretical approaches to overcome this problem, which may be divided in three types. In the case of the first type it is assumed that there exists a hidden sector in which SUSY is broken by some flavor blind mechanism, and that SUSY breaking is mediated by flavor blind interactions to the MSSM sector [1, 9, 10, 11]. The idea of the second type to overcome the SUSY flavor problem is to use the infrared attractive force of gauge interactions 12, 13, 14, 15, 16, 17, 18, 19, 20, 21]. It has been found in 18, 19, 20, 21] that thanks to the power-running law of gauge couplings 22, 23, 24] in extra dimensions [25, 26], the infrared attractiveness of running SSB parameters [27] can be so amplified that at a compactification scale the SSB terms align themselves out of their anarchical disorder at a cutoff scale $\Lambda$, even if the ratio of the cutoff scale $\Lambda$ to the compactification scale $\Lambda_{C}$ is small $\sim O\left(10^{3}\right)$. The third mechanism is based on a flavor symmetry principle [28, 29, 30, 31, 32]. In this approach one should be aware of the fact that, if a flavor symmetry is hardly broken at low energies e.g. in the Yukawa sector, then these interactions can induce non-symmetric SSB terms. In 32] it has been shown that a spontaneously broken continuous horizontal symmetry based on $S U(3)$ [33] can significantly suppress FCNCs and CP violating phases. An advantage of a discrete flavor symmetry is that no Nambu-Goldstone bosons can occur when it is spontaneously broken. In [29, 31] it has been found that a discrete flavor symmetry based on $S_{3}$ at low energy can considerably soften the SUSY flavor problem. 
In this paper, we are motivated by the desire to combine the second and third types of mechanism to soften the SUSY flavor problem. That is, in this double suppression mechanism, asymptotically free gauge interactions in extra dimensions bring a large disorder of the SSB parameters at the cutoff scale $\Lambda$ down to $O(1)$ disorder at the compactification scale $\Lambda_{C}$, and FCNCs and CP phases, which are induced by the SSB parameters in $O(1)$ disorder at low energy, are suppressed due to an intact flavor symmetry. By $O(1)$ disorder of the SSB parameters we mean e.g. $\left(m_{\max }^{2}-m_{\min }^{2}\right) / m_{\mathrm{av}}^{2} \sim O(1)$, where $m_{\mathrm{max}, \min , \text { av }}^{2}$ are the maximal, minimal and average squared soft scalar masses, respectively, and similarly for the other SSB parameters.

In Sect. II we start by considering a minimal supersymmetric extension of the $S_{3}$ invariant SM of [34, 35]. In 31], such an extension has been indeed made. There, additional $S U(2)_{L} \times$ $U(1)_{Y}$ singlet Higgs multiplets have been introduced to avoid the appearance of pseudo Nambu-Goldstone bosons. Here we would like to propose an alternative way to avoid the appearance of the pseudo Nambu-Goldstone bosons. We assume that $S_{2}$ is softly broken by certain $B$ terms in the SSB sector. It turns out that it is possible to make all the Higgses except for one very heavy without running into the problem of triviality.

In Sect. III we discuss the third type of suppression mechanism. We explicitly compute $\delta_{L L, R R, L R}^{q, \ell}$ of [7] in terms of the $S_{3}$ invariant SSB terms, and find that these $\delta$ 's can satisfy the experimental constraints if the disorder of the SSB parameters at $M_{\mathrm{SUSY}}$ is at most about one. The analysis of this section is basically a reanalysis of [31]. We, however, use a new set of input parameters in the quark sector to obtain a better agreement with the experimental results on the Cabibbo-Kobayashi-Maskawa mixing matrix $V_{\mathrm{CKM}}$.

Extra dimensions are introduced in Sect. IV. Our model is a simple extension into $\delta$ extra dimensions along the line of [23, 25, 26], in which it is assumed that the matter multiplets and the $U(1)_{Y}$ gauge multiplet are located at a fixed point, while the $S U(2)_{L}$ and $S U(3)_{C}$ gauge multiplets propagate in the bulk.

Sect. $\mathrm{V}$ is devoted to conclusion, and in Appendix we give renormalization group (RG) functions that are used in this paper. 


\section{II. $S_{3}$ INVARIANT EXTENSION OF THE MSSM}

Flavor symmetries based on a permutation symmetry have been considered by many authors in the past ${ }^{1}$. Phenomenologically viable models based on non-abelian discrete flavor symmetries $S_{3}, D_{4}$ and $A_{4}$ and also on a product of abelian discrete symmetries have been recently constructed in [34, 35], 43], [40, 41, 42] and 44, 48], respectively, which can naturally explain a large neutrino mixing. (See also [45, 46, 47, 48, 49]. ) In this section we would like to consider a supersymmetric extension of the $S_{3}$ invariant model of [34, 35].

\section{A. $S_{3}$ invariant superpotential}

Three generations of the quarks and leptons belong to the reducible representation of $S_{3}$ $\mathbf{3}=\mathbf{1}+\mathbf{2}$, respectively. They are denoted by $Q_{I}, Q_{3}, U_{I R}, U_{3 R}, D_{I R}, D_{3 R}, L_{I}, L_{3}, E_{I R}$, $E_{3 R}, N_{I R}, N_{3 R}$ in an obvious notation. We also introduce an $S_{3}$ doublet Higgs pair, $H_{I}^{U}, H_{I}^{D}(I=1,2)$, as well as an $S_{3}$ singlet Higgs pair, $H_{3}^{U}, H_{3}^{D}$. The same R-parity is assigned to these fields as in the MSSM. Then we assume that the superpotential, $W=W_{D}+W_{U}+W_{E}+W_{N}+W_{M}+W_{H}$, is invariant under $S_{3}$ permutations. Each part is given explicitly as follows:

$$
\begin{aligned}
W_{D}= & Y_{1}^{D} Q_{I} H_{3}^{D} D_{I R}+Y_{3}^{D} Q_{3} H_{3}^{D} D_{3 R} \\
& +Y_{2}^{D} f_{I J K} Q_{I} H_{J}^{D} D_{K R} \\
& +Y_{4}^{D} Q_{3} H_{I}^{D} D_{I R}+Y_{5}^{D} Q_{I} H_{I}^{D} D_{3 R}, \\
W_{U}= & Y_{1}^{U} Q_{I} H_{3}^{U} U_{I R}+Y_{3}^{U} Q_{3} H_{3}^{U} U_{3 R} \\
& +Y_{2}^{U} f_{I J K} Q_{I} H_{J}^{U} U_{K R} \\
& +Y_{4}^{U} Q_{3} H_{I}^{U} U_{I R}+Y_{5}^{U} Q_{I} H_{I}^{U} U_{3 R},
\end{aligned}
$$

\footnotetext{
${ }^{1}$ One of the first papers on permutation symmetries are [36, 37, 38]. See [35] for a partial list of references, and 39] for a review. By a flavor symmetry we mean a symmetry of the Yukawa interactions, because a symmetry of a mass matrix is not necessarily a symmetry of the theory.
} 


$$
\begin{aligned}
W_{E}= & Y_{1}^{E} L_{I} H_{3}^{D} E_{I R}+Y_{3}^{E} L_{3} H_{3}^{D} E_{3 R} \\
& +Y_{2}^{E} f_{I J K} L_{I} H_{J}^{D} E_{K R} \\
& +Y_{4}^{E} L_{3} H_{I}^{D} E_{I R}+Y_{5}^{E} L_{I} H_{I}^{D} E_{3 R}, \\
W_{N}= & Y_{1}^{N} L_{I} H_{3}^{U} N_{I R}+Y_{3}^{N} L_{3} H_{3}^{U} N_{3 R} \\
& +Y_{2}^{N} f_{I J K} L_{I} H_{J}^{U} N_{K R} \\
& +Y_{4}^{N} L_{3} H_{I}^{U} N_{I R}+Y_{5}^{N} L_{I} H_{I}^{U} N_{3 R}, \\
W_{M}= & \frac{1}{2} M_{1} N_{I R} N_{I R}+\frac{1}{2} M_{3} N_{3 R} N_{3 R}, \\
W_{H}= & \mu_{1} H_{I}^{U} H_{I}^{D}+\mu_{3} H_{3}^{U} H_{3}^{D},
\end{aligned}
$$

where

$$
f_{121}=f_{211}=f_{112}=-f_{222}=1, f_{111}=f_{221}=f_{122}=f_{212}=0 .
$$

( $f_{I J K}$ is completely symmetric). $W$ is the most general renormalizable superpotential that is $S_{3} \times R$ invariant.

\section{B. Soft supersymmetry breaking sector}

(i) Gaugino masses:

The gaugino masses are the same as in the MSSM.

(ii) Trilinear couplings:

The trilinear couplings $h$ 's can be read off from $W_{U, D, E, N}$. We denote them by $h_{I}^{U}$ etc. By symmetry, the trilinear couplings have exactly the same structure as the Yukawa couplings.

(ii) Soft scalar masses:

$S_{3}$ invariant soft scalar masses are diagonal, and have the general structure:

$$
\tilde{m}_{1}^{2} \hat{\phi}_{I}^{*} \hat{\phi}_{I}+\tilde{m}_{3}^{2} \hat{\phi}_{3}^{*} \hat{\phi}_{3}
$$

for all scalar components $\hat{\phi}$. Those of the MSSM are denoted by $m_{Q_{I}}^{2}, m_{Q_{3}}^{2}, m_{U_{I R}}^{2}, m_{U_{3 R}}^{2}$ etc. (iii) B-terms:

$S_{3}$ invariant B-terms are

$$
\mathcal{L}_{B}=B_{1}\left(\hat{H}_{1}^{U} \hat{H}_{1}^{D}+\hat{H}_{2}^{U} \hat{H}_{2}^{D}\right)+B_{3}\left(\hat{H}_{3}^{U} \hat{H}_{3}^{D}\right)+\text { h.c. }
$$


Given the superpotential (11)-(6) along with the SSB sector, we can now write down the scalar potential. For simplicity we assume that only the neutral scalar components of the Higgs supermultiplets acquire VEVs. The relevant part of the scalar potential is then given by

$$
\begin{aligned}
V= & \left(\left|\mu_{1}\right|^{2}+m_{H_{1}^{U}}^{2}\right)\left(\left|\hat{H}_{1}^{0 U}\right|^{2}+\left|\hat{H}_{2}^{0 U}\right|^{2}\right)+\left(\left|\mu_{1}\right|^{2}+m_{H_{1}^{D}}^{2}\right)\left(\left|\hat{H}_{1}^{0 D}\right|^{2}+\left|\hat{H}_{2}^{0 D}\right|^{2}\right) \\
& +\left(\left|\mu_{3}\right|^{2}+m_{H_{3}^{U}}^{2}\right)\left(\left|\hat{H}_{3}^{0 U}\right|^{2}\right)+\left(\left|\mu_{3}\right|^{2}+m_{H_{3}^{D}}^{2}\right)\left(\left|\hat{H}_{3}^{0 D}\right|^{2}\right) \\
& +\frac{1}{8}\left(\frac{3}{5} g_{1}^{2}+g_{2}^{2}\right)\left(\left|\hat{H}_{1}^{0 U}\right|^{2}+\left|\hat{H}_{2}^{0 U}\right|^{2}+\left|\hat{H}_{3}^{0 U}\right|^{2}-\left|\hat{H}_{1}^{0 D}\right|^{2}-\left|\hat{H}_{2}^{0 D}\right|^{2}-\left|\hat{H}_{3}^{0 D}\right|^{2}\right)^{2} \\
& -\left[B_{1}\left(\hat{H}_{1}^{0 U} \hat{H}_{1}^{0 D}+\hat{H}_{2}^{0 U} \hat{H}_{2}^{0 D}\right)+B_{3}\left(\hat{H}_{3}^{0 U} \hat{H}_{3}^{0 D}\right)+\text { h.c. }\right]
\end{aligned}
$$

As one can see easily, the scalar potential $V$ (10) has a continuous global symmetry $S U(2) \times U(1)$ in addition to the local electroweak gauge symmetry $S U(2)_{L} \times U(1)_{Y}$. As a result, there will be a number of pseudo Goldstone bosons that are phenomenologically unacceptable. This is a consequence of $S_{3}$ symmetry. (A similar consequence exists also in nonsupersymmetric case, too.) Therefore, we have to break $S_{3}$ symmetry explicitly. We would like to break it as soft as possible to preserve predictions from $S_{3}$ symmetry, while breaking the global $S U(2) \times U(1)$ symmetry completely. Surprisingly, there is a unique choice for a set of soft $S_{3}$ breaking terms: The softest terms in the present case have the canonical dimension two, implying they should be in the SSB sector. As for the soft scalar masses, we have an important consequence (8) from $S_{3}$ symmetry that they are diagonal in generations. Since we would like to preserve this structure, the only choice is to introduce the soft $S_{3}$ breaking terms in the B sector (9). Moreover, looking at the $S_{3}$ invariant scalar potential $V$ (10), we observe that it has an abelian discrete symmetry

$$
S_{2}^{\prime}: \hat{H}_{1}^{U, D} \leftrightarrow \hat{H}_{2}^{U, D}
$$

We assume that the soft $S_{3}$ breaking terms respect this discrete symmetry (11), and add the following soft $S_{3}$ breaking Lagrangian:

$$
\mathcal{L}_{S_{3} B}=B_{4}\left(\hat{H}_{1}^{U} \hat{H}_{2}^{D}+\hat{H}_{2}^{U} \hat{H}_{1}^{D}\right)+B_{5} \hat{H}_{3}^{U}\left(\hat{H}_{1}^{D}+\hat{H}_{2}^{D}\right)+B_{6} \hat{H}_{3}^{D}\left(\hat{H}_{1}^{U}+\hat{H}_{2}^{U}\right)+\text { h.c. }
$$

The resulting scalar potential can be analyzed, and one finds that a local minimum respecting $S_{2}^{\prime}$ symmetry, i.e.

$$
\begin{aligned}
& <\hat{H}_{1}^{U}>=<\hat{H}_{2}^{U}>=v_{U} / 2 \neq 0, \quad<\hat{H}_{1}^{D}>=<\hat{H}_{2}^{D}>=v_{D} / 2 \neq 0 \\
& <\hat{H}_{3}^{U}>=v_{3 U} / \sqrt{2} \neq 0, \quad<\hat{H}_{3}^{D}>=v_{3 D} / \sqrt{2} \neq 0
\end{aligned}
$$


can occur. We find the the lightest Higgs, the SM Higgs, can be written as a linear combination

$$
h_{\mathrm{SM}}=\left(v_{D} \hat{H}_{+}^{0 D}+v_{3 D} \hat{H}_{3}^{0 D}+v_{U} \hat{H}_{+}^{0 U}+v_{3 U} \hat{H}_{3}^{0 U}\right) / v
$$

where

$$
\hat{H}_{+}^{0 D, U}=\frac{1}{\sqrt{2}}\left(\hat{H}_{1}^{0 D, U}+\hat{H}_{2}^{0 D, U}\right), v=\left(v_{U}^{2}+v_{3 U}^{2}+v_{D}^{2}+v_{3 D}^{2}\right)^{1 / 2} \simeq 246 \mathrm{GeV}
$$

Its mass is approximately given by

$$
m_{h} \simeq\left((3 / 5) g_{1}^{2}+g_{2}^{2}\right)\left(v_{U}^{2}+v_{3 U}^{2}-v_{D}^{2}-v_{3 D}^{2}\right)^{2} / v
$$

for $\mu^{2 \prime} s, B^{\prime} s>>v^{2}$. It can be shown that the masses of other Higgs multiplets can be made arbitrarily heavy. From (16) we see that the tree-level upper bound for $m_{h}$ is exactly the same as in the MSSM.

Because of the very nature of the SSB terms, the explicit breaking of $S_{3}$ in the B terms (12) does not propagate to the other sector in the sense that it does not produce $S_{3}$ violating infinities in other sectors. Furthermore, although the superpotential (11)-(6) and the corresponding trilinear couplings do not respect the $S_{2}^{\prime}$ symmetry (11), they can not generate $S_{2}^{\prime}$ violating infinite B terms because they can generate only $S_{3}$ invariant terms in the Higgs sector, which are however automatically $S_{2}^{\prime}$ invariant.

\section{C. $Z_{2}$ symmetry in the leptonic sector, mass matrices and diagonalization}

As in [34, 35] we assume the existence of an abelian discrete symmetry $Z_{2}$ in the leptonic sector. The $Z_{2}$ parity assignment is:

$$
+\quad \text { for } H_{I}^{U, D}, L_{3}, L_{I}, E_{3 R}, E_{I R}, N_{I R} \text { and }- \text { for } H_{3}^{U, D}, N_{3 R} \text {, }
$$

which kills

$$
Y_{1}^{E}, Y_{3}^{E}, Y_{1}^{N}, \text { and } Y_{5}^{N}
$$

in the Yukawa sector, and

$$
h_{1}^{E}, h_{3}^{E}, h_{1}^{N}, \text { and } h_{5}^{N}
$$


in the SSB sector. Note that this symmetry is explicitly broken in the quark sector. However, the $F$ term non-renormalization theorem prevents from producing $Z_{2}$ violating terms in the leptonic sector. As a result of (13), the quark and lepton mass matrices take the general form

$$
\mathbf{M}_{a}=\left(\begin{array}{ccc}
m_{1}^{a}+m_{2}^{a} & m_{2}^{a} & m_{5}^{a} \\
m_{2}^{a} & m_{1}^{a}-m_{2}^{a} & m_{5}^{a} \\
m_{4}^{a} & m_{4}^{a} & m_{3}^{a}
\end{array}\right) \text { with } m_{1}^{e}=m_{3}^{e}=m_{1}^{\nu}=m_{5}^{\nu}=0
$$

where $a=u, d, e, \nu$. It has been found in [34] that the mass matrices of the general form (20) are consistent with all the observed quark and lepton masses and mixing angles. We emphasize that this result can remain valid after supersymmetrization of the model.

Next we consider diagonalization of the mass matrices, and start with the charged lepton mass matrix $\mathbf{M}_{e}$. It has only real parameters, and they are given by

$$
m_{2}^{e} \simeq 0.07474 \mathrm{GeV}, m_{4}^{e} \simeq 0.0005141 \mathrm{GeV}, m_{5}^{e} \simeq 1.254 \mathrm{GeV}, m_{1}^{e}=m_{3}^{e}=0
$$

Then the unitary matrices $U_{e L}$ and $U_{e R}$ defined as

$$
U_{e L}^{\dagger} \mathbf{M}_{e} U_{e R}=\operatorname{diag}\left(m_{e}, m_{\mu}, m_{\tau}\right)
$$

are found to be

$$
\begin{aligned}
& U_{e L} \simeq\left(\begin{array}{ccc}
-0.003452 & -0.7071 & 0.7071 \\
0.003427 & 0.7071 & 0.7071 \\
1.0 & -0.004864 & 0.00001722
\end{array}\right), \\
& U_{e R} \simeq\left(\begin{array}{ccc}
0.9982 & -0.000023578 & 0.05949 \\
0.00002362 & -1.0 & 0 \\
-0.05949 & 1.4 \times 10^{-6} & 0.9982
\end{array}\right) .
\end{aligned}
$$

Note that the maximal mixing appearing in $U_{e L}$ is responsible for the atmospheric neutrino mixing, while the solar neutrino mixing is explained by the large mixing in the rotation matrix of the neutrino mass matrix ${ }^{2}$. That is, the elements, $\left(U_{e L}\right)_{21}$ and $\left(U_{e L}\right)_{23}$ become, respectively, the $(1,3)$ and $(3,3)$ elements of the neutrino mixing matrix $V_{\text {MNS; }}$;

$$
U_{e 3} \simeq-0.0034, \cos \theta_{\mathrm{atm}}=1 / \sqrt{2}
$$

\footnotetext{
${ }^{2}$ See [50] for recent developments on neutrino physics.
} 
As in the leptonic case, we introduce unitary matrices $U_{u(d) L}$ and $U_{u(d) R}$ satisfying

$$
U_{u(d) L}^{\dagger} \mathbf{M}_{u(d)} U_{u(d) R}=\operatorname{diag}\left(m_{u(d)}, m_{c(s)}, m_{t(b)}\right) .
$$

Realistic quark masses as well as CKM matrix $V_{\mathrm{CKM}}$ can be obtained from ${ }^{3}$

$$
\begin{aligned}
& m_{1}^{u}=-0.06504 \mathrm{GeV}, m_{2}^{u}=-0.06148 \mathrm{GeV}, m_{3}^{u}=173.5 \mathrm{GeV}, \\
& m_{4}^{u}=10.70 \mathrm{GeV}, m_{5}^{u}=4.166 \mathrm{GeV} \\
& m_{1}^{d}=0.008974 \mathrm{GeV}, m_{2}^{d}=0.01460 \mathrm{GeV}, m_{3}^{d}=1.950+1.548 I \mathrm{GeV}, \\
& m_{4}^{d}=1.045 \mathrm{GeV}, m_{5}^{d}=0.1427 \mathrm{GeV},
\end{aligned}
$$

where we have assumed that only $m_{3}^{d}$ is complex. The values given in (27) are not a unique choice. However, numerical analyses show that the values can not be continuously deformed without changing the values of the quark masses and $V_{\text {CKM }}$ (under the assumption that only $m_{3}^{d}$ is complex). The unitary matrices for the set of the parameter values (27) are given by

$$
\begin{gathered}
U_{u L} \simeq\left(\begin{array}{ccc}
0.6366 & -0.7709 & 0.02375 \\
-0.7712 & -0.6361 & 0.02379 \\
0.003233 & 0.03346 & 0.9994
\end{array}\right), \\
U_{u R} \simeq\left(\begin{array}{ccc}
0.6365 & 0.7688 & 0.06136 \\
-0.7712 & 0.6336 & 0.06138 \\
0.008306 & -0.08639 & 0.9962
\end{array}\right),
\end{gathered}
$$

\footnotetext{
${ }^{3}$ The values of the input parameters used here are slightly different from those in [31, 34]. They yield $V_{\mathrm{CKM}}$ in a better agreement with the experimental values.
} 


$$
\begin{aligned}
& U_{d L} \simeq\left(\begin{array}{c}
-0.7497,0.549,0.04444 \\
0.6284,0.7409,0.0410 \\
0.01124,-0.06039,0.9424
\end{array}\right) \\
&+I\left(\begin{array}{c}
0.2037,-0.3043,-0.01238 \\
-0.03858,-0.2299,-0.01357 \\
-0.00321,-0.01458,0.3285
\end{array}\right), \\
& U_{d R}=\left(\begin{array}{c}
0.74582,-0.4371,0.3400 \\
-0.6277,-0.6584,0.3396 \\
-0.08189,0.4862,0.8129
\end{array}\right) \\
&+I\left(\begin{array}{c}
0.2027,-0.2863,0.1182 \\
-0.03772,-0.2043,0.1183 \\
-0.02341,-0.1238,-0.2834
\end{array}\right) .
\end{aligned}
$$

Note that the off-diagonal elements in $U_{d L}$ and $U_{d R}$ carry large complex phases. For completeness we write the explicit values of $V_{\mathrm{CKM}}$ and the quark masses for the set of parameters (27):

$$
\left|V_{\mathrm{CKM}}\right|=\left|U_{u L}^{\dagger} U_{d L}\right| \simeq\left(\begin{array}{c}
0.975,0.223,0.0037 \\
0.222,0.974,0.041 \\
0.0084,0.040,0.999
\end{array}\right)
$$

with

$$
J \simeq 3.0 \times 10^{-3}, \phi_{1}(\beta) \simeq 23^{\circ}, \phi_{3}(\gamma) \simeq 67^{\circ}
$$

and

$$
\begin{aligned}
& m_{d} \simeq 4.4 \mathrm{MeV}, m_{s} \simeq 0.09 \mathrm{GeV}, m_{b} \simeq 2.9 \mathrm{GeV} \\
& m_{u} \simeq 2.3 \mathrm{MeV}, m_{c} \simeq 0.64 \mathrm{GeV}, m_{t} \simeq 174 \mathrm{GeV}
\end{aligned}
$$

where $J$ is the Jarlskog invariant. 


\section{SUPPRESSION OF FCNCS AT $M_{\mathrm{SUSY}}$}

Since all the soft scalar masses have the form (요 $)$, we write the mass matrices as

$$
\tilde{\mathbf{m}}_{a L L}^{2}=m_{a}^{2}\left(\begin{array}{ccc}
a_{L}^{a} & 0 & 0 \\
0 & a_{L}^{a} & 0 \\
0 & 0 & b_{L}^{a}
\end{array}\right), \tilde{\mathbf{m}}_{a R R}^{2}=m_{a}^{2}\left(\begin{array}{ccc}
a_{R}^{a} & 0 & 0 \\
0 & a_{R}^{a} & 0 \\
0 & 0 & b_{R}^{a}
\end{array}\right) \quad(a=\tilde{\ell}, \tilde{q})
$$

where $m_{\tilde{\ell}, \tilde{q}}$ denote the average of the slepton and squark masses, respectively, and $\left(a_{L(R)}, b_{L(R)}\right)$ are dimensionless free parameters of $O(1)$. Further, since the trilinear interactions are also $S_{3}$ invariant, the left-right mass matrix can be written as

$$
\tilde{\mathbf{m}}_{a L R}^{2}=\left(\begin{array}{ccc}
m_{1}^{a} A_{1}^{a}+m_{2}^{a} A_{2}^{a} & m_{2}^{a} A_{2}^{a} & m_{5}^{a} A_{5}^{a} \\
m_{2}^{a} A_{2}^{a} & m_{1}^{a} A_{1}^{a}-m_{2}^{a} A_{2}^{a} & m_{5}^{a} A_{5}^{a} \\
m_{4}^{a} A_{4}^{a} & m_{4}^{a} A_{4}^{a} & m_{3}^{a} A_{3}^{a}
\end{array}\right) \quad(a=\tilde{\ell}, \tilde{q})
$$

where $A_{i}^{a}$ are free parameters of dimension one. Here we assume that they are in the same order as the gaugino masses.

We consider FCNC processes, e.g. $\operatorname{Br}(\mu \rightarrow e+\gamma)$, that are proportional to the offdiagonal elements of

$$
\Delta_{L L, R R}^{a}=U_{a L, R}^{\dagger} \tilde{\mathbf{m}}_{a L L, R R}^{2} U_{a L, R} \text { and } \Delta_{L R}^{a}=U_{a L}^{\dagger} \tilde{\mathbf{m}}_{a L R}^{2} U_{a R}
$$

By using the unitary matrices given in Eqs. (23), (24) and (28)-(31), $\Delta$ 's can be explicitly evaluated. In [7], experimental bounds on the dimensionless quantities

$$
\delta_{L L, R R, L R}^{a}=\Delta_{L L, R R, L R}^{a} / m_{\tilde{a}}^{2} \quad(a=\ell, q),
$$

are given, which are summarized in Table I. The theoretical values of $\delta$ 's for the present model are calculated below, where

$$
\Delta a_{L, R}^{a}=a_{L, R}^{a}-b_{L, R}^{a}, \quad \tilde{A}_{i}^{a}=\frac{A_{i}^{a}}{m_{\tilde{a}}}(a=\ell, q),
$$

and $a_{L, R}, b_{L, R}$ are defined in (35). 
Leptonic sector $(L L$ and $R R)$ :

$$
\begin{aligned}
\left(\delta_{12}^{\ell}\right)_{L L} & \simeq\left(\delta_{21}^{\ell}\right)_{L L} \simeq 4.8 \times 10^{-3} \Delta a_{L}^{\ell}, \\
\left(\delta_{13}^{\ell}\right)_{L L} & \simeq\left(\delta_{31}^{\ell}\right)_{L L} \simeq-1.7 \times 10^{-5} \Delta a_{L}^{\ell}, \\
\left(\delta_{23}^{\ell}\right)_{L L} & \simeq\left(\delta_{32}^{\ell}\right)_{L L} \simeq 8.4 \times 10^{-8} \Delta a_{L}^{\ell}, \\
\left(\delta_{12}^{\ell}\right)_{R R} & \simeq\left(\delta_{21}^{\ell}\right)_{R R} \simeq 8.4 \times 10^{-8} \Delta a_{R}^{\ell}, \\
\left(\delta_{13}^{\ell}\right)_{R R} & \simeq\left(\delta_{31}^{\ell}\right)_{R R} \simeq 5.9 \times 10^{-2} \Delta a_{R}^{\ell}, \\
\left(\delta_{23}^{\ell}\right)_{R R} & \simeq\left(\delta_{32}^{\ell}\right)_{R R} \simeq-1.4 \times 10^{-6} \Delta a_{R}^{\ell} .
\end{aligned}
$$

Leptonic sector $(L R)$ :

$$
\begin{aligned}
\operatorname{Im}\left(\delta_{i j}^{\ell}\right)_{L R} & =0, \\
\left(\delta_{12}^{\ell}\right)_{L R} & \simeq 5.1 \times 10^{-6}\left(\tilde{A}_{2}^{\ell}-\tilde{A}_{4}^{\ell}\right)\left(\frac{100 \mathrm{GeV}}{m_{\tilde{\ell}}}\right), \\
\left(\delta_{21}^{\ell}\right)_{L R} & \simeq 2.5 \times 10^{-8}\left(\tilde{A}_{2}^{\ell}-\tilde{A}_{4}^{\ell}\right)\left(\frac{100 \mathrm{GeV}}{m_{\tilde{\ell}}}\right), \\
\left(\delta_{13}^{\ell}\right)_{L R} & \simeq 3.1 \times 10^{-7}\left(\tilde{A}_{4}^{\ell}-\tilde{A}_{5}^{\ell}\right)\left(\frac{100 \mathrm{GeV}}{m_{\tilde{\ell}}}\right), \\
\left(\delta_{31}^{\ell}\right)_{L R} & \simeq 1.1 \times 10^{-3}\left(\tilde{A}_{2}^{\ell}-\tilde{A}_{5}^{\ell}\right)\left(\frac{100 \mathrm{GeV}}{m_{\tilde{\ell}}}\right), \\
\left(\delta_{23}^{\ell}\right)_{L R} & \simeq-1.5 \times 10^{-9}\left(\tilde{A}_{4}^{\ell}-\tilde{A}_{5}^{\ell}\right)\left(\frac{100 \mathrm{GeV}}{m_{\tilde{\ell}}}\right), \\
\left(\delta_{32}^{\ell}\right)_{L R} & \simeq-2.5 \times 10^{-8}\left(\tilde{A}_{2}^{\ell}-\tilde{A}_{5}^{\ell}\right)\left(\frac{100 \mathrm{GeV}}{m_{\tilde{\ell}}}\right) .
\end{aligned}
$$

$\underline{\text { Up quark sector }(L L \text { and } R R) \text { : }}$

$$
\begin{aligned}
& \left(\delta_{12}^{u}\right)_{L L} \simeq\left(\delta_{21}^{d}\right)_{L L} \simeq-1.1 \times 10^{-4} \Delta a_{L}^{u}, \\
& \left(\delta_{13}^{u}\right)_{L L} \simeq\left(\delta_{31}^{d}\right)_{L L} \simeq-3.2 \times 10^{-3} \Delta a_{L}, \\
& \left(\delta_{23}^{u}\right)_{L L} \simeq\left(\delta_{32}^{d}\right)_{L L} \simeq-3.3 \times 10^{-2} \Delta a_{L}^{u}, \\
& \left(\delta_{12}^{u}\right)_{R R} \simeq\left(\delta_{21}^{u}\right)_{R R} \simeq 7.2 \times 10^{-4} \Delta a_{R}^{u}, \\
& \left(\delta_{13}^{u}\right)_{R R} \simeq\left(\delta_{31}^{u}\right)_{R R} \simeq-8.2 \times 10^{-3} \Delta a_{R}^{u}, \\
& \left(\delta_{23}^{u}\right)_{R R} \simeq\left(\delta_{32}^{u}\right)_{R R} \simeq 8.6 \times 10^{-2} \Delta a_{R}^{u} .
\end{aligned}
$$




\section{Up quark sector $(L R)$ :}

$$
\begin{aligned}
& \left(\delta_{11}^{u}\right)_{L R} \simeq\left[-1.3 \times 10^{-4} \tilde{A}_{1}^{u}+1.4 \times 10^{-4} \tilde{A}_{2}^{u}\right]\left(\frac{500 \mathrm{GeV}}{m_{\tilde{q}}}\right) \\
& \left(\delta_{12}^{u}\right)_{L R} \simeq-\left(\delta_{21}^{u}\right)_{L R} \simeq-9.7 \times 10^{-5}\left(\tilde{A}_{2}^{u}+\tilde{A}_{3}^{u}-\tilde{A}_{4}^{u}-\tilde{A}_{5}^{u}\right)\left(\frac{500 \mathrm{GeV}}{m_{\tilde{q}}}\right), \\
& \left(\delta_{13}^{u}\right)_{L R} \simeq 1.1 \times 10^{-3}\left(\tilde{A}_{3}^{u}-\tilde{A}_{5}^{u}\right)\left(\frac{500 \mathrm{GeV}}{m_{\tilde{q}}}\right), \\
& \left(\delta_{31}^{u}\right)_{L R} \simeq 2.9 \times 10^{-3}\left(\tilde{A}_{3}^{u}-\tilde{A}_{4}^{u}\right)\left(\frac{500 \mathrm{GeV}}{m_{\tilde{q}}}\right), \\
& \left(\delta_{23}^{u}\right)_{L R} \simeq 1.2 \times 10^{-2}\left(\tilde{A}_{3}^{u}-\tilde{A}_{5}^{u}\right)\left(\frac{500 \mathrm{GeV}}{m_{\tilde{q}}}\right), \\
& \left(\delta_{32}^{u}\right)_{L R} \simeq-3.0 \times 10^{-2}\left(\tilde{A}_{3}^{u}-\tilde{A}_{4}^{u}\right)\left(\frac{500 \mathrm{GeV}}{m_{\tilde{q}}}\right) .
\end{aligned}
$$

$\underline{\text { Down quark sector }(L L \text { and } R R) \text { : }}$

$$
\begin{aligned}
& \left(\delta_{12}^{d}\right)_{L L} \simeq\left(-6.1 \times 10^{-4}-3.8 \times 10^{-4} I\right) \Delta a_{L}^{d}, \\
& \left(\delta_{21}^{d}\right)_{L L} \simeq\left(-6.1 \times 10^{-4}+3.8 \times 10^{-4} I\right) \Delta a_{L}^{d}, \\
& \left(\delta_{13}^{d}\right)_{L L} \simeq\left(1.1 \times 10^{-2}+1.5 \times 10^{-4} I\right) \Delta a_{L}^{d}, \\
& \left(\delta_{31}^{d}\right)_{L L} \simeq\left(1.1 \times 10^{-2}-1.5 \times 10^{-4} I\right) \Delta a_{L}^{d}, \\
& \left(\delta_{23}^{d}\right)_{L L} \simeq\left(5.3 \times 10^{-2}-3.2 \times 10^{-2} I\right) \Delta a_{L}^{d}, \\
& \left(\delta_{32}^{d}\right)_{L L} \simeq\left(5.3 \times 10^{-2}+3.2 \times 10^{-2} I\right) \Delta a_{L}^{d}, \\
& \left(\delta_{12}^{d}\right)_{R R} \simeq\left(-3.7 \times 10^{-2}+2.0 \times 10^{-2} I\right) \Delta a_{R}^{d}, \\
& \left(\delta_{21}^{d}\right)_{R R} \simeq\left(-3.7 \times 10^{-2}-2.0 \times 10^{-2} I\right) \Delta a_{R}^{d}, \\
& \left(\delta_{13}^{d}\right)_{R R} \simeq\left(-2.6 \times 10^{-2}+6.9 \times 10^{-2} I\right) \Delta a_{R}^{d}, \\
& \left(\delta_{31}^{d}\right)_{R R} \simeq\left(-2.6 \times 10^{-2}-6.9 \times 10^{-2} I\right) \Delta a_{R}^{d}, \\
& \left(\delta_{23}^{d}\right)_{R R} \simeq\left(-3.2 \times 10^{-1}+2.9 \times 10^{-1} I\right) \Delta a_{R}^{d}, \\
& \left(\delta_{32}^{d}\right)_{R R} \simeq\left(-3.2 \times 10^{-1}-2.9 \times 10^{-1} I\right) \Delta a_{R}^{d} .
\end{aligned}
$$


Down quark sector $(L R)$ :

$$
\begin{aligned}
\left(\delta_{11}^{d}\right)_{L R} \simeq & {\left[-1.6 \tilde{A}_{1}^{d}+2.3 \tilde{A}_{2}^{d}-I 0.6\left(\tilde{A}_{1}^{d}-\tilde{A}_{2}^{u}\right)+I 0.5\left(\tilde{A}_{3}^{d}+\tilde{A}_{2}^{d}-\tilde{A}_{4}^{d}-\tilde{A}_{5}^{d}\right)\right] } \\
& \times 10^{-5}\left(\frac{500 \mathrm{GeV}}{m_{\tilde{q}}}\right), \\
\left(\delta_{12}^{d}\right)_{L R} \simeq & {\left[(2.4+1.7 I) \times 10^{-5}\left(\tilde{A}_{2}^{d}-\tilde{A}_{5}^{d}\right)+(2.2+1.9 I) \times 10^{-5}\left(\tilde{A}_{3}^{d}-\tilde{A}_{4}^{d}\right)\right] } \\
& \times\left(\frac{500 \mathrm{GeV}}{m_{\tilde{q}}}\right), \\
\left(\delta_{21}^{d}\right)_{L R} \simeq & {\left[(2.7+1.9 I) \times 10^{-5}\left(\tilde{A}_{2}^{d}-\tilde{A}_{5}^{d}\right)+(2.0+1.7 I) \times 10^{-5}\left(\tilde{A}_{3}^{d}-\tilde{A}_{4}^{d}\right)\right] } \\
& \times\left(\frac{500 \mathrm{GeV}}{m_{\tilde{q}}}\right), \\
\left(\delta_{13}^{d}\right)_{L R} \simeq- & {\left[(4.1+2.9 I) \times 10^{-5}\left(\tilde{A}_{3}^{d}-\tilde{A}_{5}^{d}\right)+(1.4+0.1 I) \times 10^{-5}\left(\tilde{A}_{2}^{d}-\tilde{A}_{4}^{d}\right)\right] } \\
\times & \left(\frac{500 \mathrm{GeV}}{m_{\tilde{q}}}\right), \\
\left(\delta_{31}^{d}\right)_{L R} \simeq & -(3.4+2.4 I) \times 10^{-4}\left(\tilde{A}_{3}^{d}-\tilde{A}_{4}^{d}\right)\left(\frac{500 \mathrm{GeV}}{m_{\tilde{q}}}\right), \\
\left(\delta_{23}^{d}\right)_{L R} \simeq & {\left[-2.6 \times 10^{-4}\left(\tilde{A}_{3}^{d}-\tilde{A}_{4}^{d}\right)+3.4 \times 10^{-4}\left(\tilde{A}_{4}^{d}-\tilde{A}_{5}^{d}\right)\right.} \\
& \left.+I\left(\tilde{A}_{1}^{d}+\tilde{A}_{2}^{d}-3 \tilde{A}_{3}^{d}-\tilde{A}_{4}^{d}+2 \tilde{A}_{5}^{d}\right) \times 10^{-5}\right]\left(\frac{500 \mathrm{GeV}}{m_{\tilde{q}}}\right), \\
\left(\delta_{32}^{d}\right)_{L R} \simeq & (2.5+0.2 I) \times 10^{-3}\left(\tilde{A}_{3}^{d}-\tilde{A}_{4}^{d}\right)\left(\frac{500 \mathrm{GeV}}{m_{\tilde{q}}}\right) .
\end{aligned}
$$

Comparing the $\delta$ 's above with Table I, we see that the experimental bounds for the most of the cases are satisfied, if $|\Delta a|$ 's and $\left|\left(\tilde{A}_{i}-\tilde{A}_{j}\right)\right|$ 's are less than about one. The experimental constraints coming from the $\mathrm{CP}$ violations in the $K^{0}-\bar{K}^{0}$ system on $\delta_{12}^{d}$, more precisely on $\sqrt{\left|\operatorname{Im}\left(\delta_{12}^{d}\right)_{L L, R R}^{2}\right|}, \sqrt{\left|\operatorname{Im}\left(\delta_{12}^{d}\right)_{L L}\left(\delta_{12}^{d}\right)_{R R}\right|}$ and $\left|\operatorname{Im}\left(\delta_{12}^{d}\right)_{L R}\right|$ are very severe. Note however, one of the most strong constraint coming from $\epsilon^{\prime} / \epsilon$ on $\left|\operatorname{Im}\left(\delta_{12}^{d}\right)_{L R}\right|$ is satisfied. It seems at first sight that $\left(\delta_{12}^{d}\right)_{R R}$ is too large to satisfy $\sqrt{\left|\operatorname{Im}\left(\delta_{12}^{d}\right)_{R R}^{2}\right|}<3.2 \times 10^{-3} \tilde{m}_{\tilde{q}}$, because $\left(\delta_{12}^{d}\right)_{R R} \sim O\left(10^{-2}\right) \Delta a_{R}^{d}$ in the present model as we can see from (44). Fortunately, this is not the case. First of all, all the $a$ 's are real, because the soft scalar mass matrices are diagonal by $S_{3}$ symmetry. Secondly, there is a freedom to make a phase rotation of the fermion mass eigenstates without changing their masses and $V_{\mathrm{CKM}}$. Under this phase 
rotation, the unitary matrices undergo as

$$
U_{d L, R} \rightarrow U_{d L, R} \times\left(\begin{array}{ccc}
e^{i \varphi_{1}} & 0 & 0 \\
0 & e^{i \varphi_{2}} & 0 \\
0 & 0 & e^{i \varphi_{3}}
\end{array}\right)
$$

Then $\delta_{i j}^{d}$ change to

$$
\delta_{i j}^{d} \times \exp -i\left(\varphi_{i}-\varphi_{j}\right)
$$

which means

$$
\left(\delta_{12}^{d}\right)_{R R} \rightarrow\left(-3.7 \times 10^{-2}+2.0 \times 10^{-2} I\right) \exp -i\left(\varphi_{1}-\varphi_{2}\right) \Delta a_{R}^{d}
$$

Therefore, it is always possible to make $\left(\delta_{12}^{d}\right)_{R R}$ and simultaneously $\left(\delta_{21}^{d}\right)_{R R}$ real so that the constraints on $\left|\operatorname{Im}\left(\delta_{12}^{d}\right)_{R R}^{2}\right|$ and $\left|\operatorname{Im}\left(\delta_{12}^{d}\right)_{L L}\left(\delta_{12}^{d}\right)_{R R}\right|$ can be satisfied. Note that the phase rotation (47) has practically no influence on the other constraints. The constraints from the electric dipole moment $(\mathrm{EDM})$ of the neutron on $\left|\operatorname{Im}\left(\delta_{11}^{d}\right)_{L R}\right|$ and $\left|\operatorname{Im}\left(\delta_{11}^{u}\right)_{L R}\right|$ are also very severe $^{4}$. It is not possible to make them real by the phase rotation (47). Therefore, we have to fine tune certain $A^{\prime}$ 's. Note, however, that it is possible to make $A_{2}^{q}, A_{4}^{q}$ and $A_{5}^{q}$ real by an appropriate phase rotation of the squarks. In this case we have to assume that

$$
\left|\operatorname{Im}\left(\tilde{A}_{1}^{u}\right)\right| \lesssim O\left(10^{-2}\right) \text { and }\left|\operatorname{Im}\left(\tilde{A}_{1,3}^{d}\right)\right| \lesssim O\left(10^{-1}\right)
$$

while their real parts can be $O(1)$.

From the analyses in this section, we conclude that, apart from the fine tuning (49), the FCNCs and CP phases, which are induced by the SSB parameters in $O(1)$ disorder at $M_{\mathrm{SuSY}}$, are sufficiently suppressed to satisfy the experimental constraints. This is a consequence of $S_{3}$ flavor symmetry.

\section{SUPPRESSION OF FCNCS AT THE COMPACTIFICATION SCALE}

As we have seen in the previous section, $S_{3}$ flavor symmetry can suppress FCNCs and dangerous CP violating phases at $M_{\mathrm{SUSY}}$. There, we have assumed that $\Delta a$ 's and $\tilde{A}$ 's are

\footnotetext{
${ }^{4}$ The EDM of the electron in the present model is practically zero, because all the Yukawa couplings $Y^{E}$ and also the corresponding trilinear couplings $h^{E}$ can be made real by appropriate phase rotations of the corresponding fermions and their scalar partners. A possible source for the EDM of the electron is the complex Yukawa couplings $Y_{3}^{N}$ and $Y_{4}^{N}$ in (4).
} 


\begin{tabular}{|c|c||c|c|}
\hline & Exp. bound & & Exp. bound \\
\hline$\left|\left(\delta_{12}^{\ell}\right)_{L L, R R}\right|$ & $7.7 \times 10^{-3} \tilde{m}_{\tilde{\ell}}^{2}$ & $\left|\left(\delta_{12}^{\ell}\right)_{L R}\right|$ & $1.7 \times 10^{-6} \tilde{m}_{\tilde{\ell}}^{2}$ \\
\hline$\left|\left(\delta_{13}^{\ell}\right)_{L L, R R}\right|$ & $29 \tilde{m}_{\tilde{\ell}}^{2}$ & $\left|\left(\delta_{13}^{\ell}\right)_{L R}\right|$ & $1.1 \times 10^{-1} \tilde{m}_{\tilde{\ell}}^{2}$ \\
\hline$\left|\left(\delta_{23}^{\ell}\right)_{L L, R R}\right|$ & $5.3 \tilde{m}_{\tilde{\ell}}^{2}$ & $\left|\left(\delta_{23}^{\ell}\right)_{L R}\right|$ & $2.0 \times 10^{-2} \tilde{m}_{\tilde{\ell}}^{2}$ \\
\hline$\sqrt{\left|\operatorname{Re}\left(\delta_{12}^{d}\right)_{L L, R R}^{2}\right|}$ & $4.0 \times 10^{-2} \tilde{m}_{\tilde{q}}$ & $\sqrt{\left|\operatorname{Re}\left(\delta_{12}^{d}\right)_{L L}\left(\delta_{12}^{d}\right)_{R R}\right|}$ & $2.8 \times 10^{-3} \tilde{m}_{\tilde{q}}$ \\
\hline$\sqrt{\left|\operatorname{Re}\left(\delta_{12}^{d}\right)_{L R}^{2}\right|}$ & $4.4 \times 10^{-3} \tilde{m}_{\tilde{q}}$ & $\sqrt{\left|\operatorname{Re}\left(\delta_{13}^{d}\right)_{L L, R R}^{2}\right|}$ & $9.8 \times 10^{-2} \tilde{m}_{\tilde{q}}$ \\
\hline$\sqrt{\left|\operatorname{Re}\left(\delta_{13}^{d}\right)_{L L}\left(\delta_{13}^{d}\right)_{R R}\right|}$ & $1.8 \times 10^{-2} \tilde{m}_{\tilde{q}}$ & $\sqrt{\left|\operatorname{Re}\left(\delta_{13}^{d}\right)_{L R}^{2}\right|}$ & $3.3 \times 10^{-3} \tilde{m}_{\tilde{q}}$ \\
\hline$\sqrt{\left|\operatorname{Re}\left(\delta_{12}^{u}\right)_{L L, R R}^{2}\right|}$ & $1.0 \times 10^{-1} \tilde{m}_{\tilde{q}}$ & $\sqrt{\left|\operatorname{Re}\left(\delta_{12}^{u}\right)_{L L}\left(\delta_{12}^{u}\right)_{R R}\right|}$ & $1.7 \times 10^{-2} \tilde{m}_{\tilde{q}}$ \\
\hline$\sqrt{\left|\operatorname{Re}\left(\delta_{12}^{u}\right)_{L R}^{2}\right|}$ & $3.1 \times 10^{-3} \tilde{m}_{\tilde{q}}$ & $\sqrt{\left|\operatorname{Im}\left(\delta_{12}^{d}\right)_{L L, R R}^{2}\right|}$ & $3.2 \times 10^{-3} \tilde{m}_{\tilde{q}}$ \\
\hline$\sqrt{\left|\operatorname{Im}\left(\delta_{12}^{d}\right)_{L L}\left(\delta_{12}^{d}\right)_{R R}\right|}$ & $2.2 \times 10^{-4} \tilde{m}_{\tilde{q}}$ & $\sqrt{\left|\operatorname{Im}\left(\delta_{12}^{d}\right)_{L R}^{2}\right|}$ & $3.5 \times 10^{-4} \tilde{m}_{\tilde{q}}$ \\
\hline$\left|\left(\delta_{23}^{d}\right)_{L L, R R}\right|$ & $8.2 \tilde{m}_{\tilde{q}}^{2}$ & $\left|\left(\delta_{23}^{d}\right)_{L R}\right|$ & $1.6 \times 10^{-2} \tilde{m}_{\tilde{q}}^{2}$ \\
\hline$\left|\operatorname{Im}\left(\delta_{12}^{d}\right)_{L L, R R}\right|$ & $4.8 \times 10^{-1} \tilde{m}_{\tilde{q}}^{2}$ & $\left|\operatorname{Im}\left(\delta_{12}^{d}\right)_{L R}\right|$ & $2.0 \times 10^{-5} \tilde{m}_{\tilde{q}}^{2}$ \\
\hline$\left|\operatorname{Im}\left(\delta_{11}^{d}\right)_{L R}\right|$ & $3.0 \times 10^{-6} \tilde{m}_{\tilde{q}}^{2}$ & $\left|\operatorname{Im}\left(\delta_{11}^{u}\right)_{L R}\right|$ & $5.9 \times 10^{-6} \tilde{m}_{\tilde{q}}^{2}$ \\
\hline$\left|\operatorname{Im}\left(\delta_{11}^{\ell}\right)_{L R}\right|$ & $3.7 \times 10^{-7} \tilde{m}_{\tilde{\ell}}^{2}$ & & \\
\hline
\end{tabular}

TABLE I: Experimental bounds on $\delta$ 's, where the parameters $\tilde{m}_{\tilde{\ell}}$ and $\tilde{m}_{\tilde{q}}$ denote $m_{\tilde{\ell}} / 100 \mathrm{GeV}$ and $m_{\tilde{\ell}} / 500 \mathrm{GeV}$, respectively. See [7] for details.

$O(1)$ at $M_{\text {SUSY }}($ they are defined in (39)). At a more fundamental scale, they may be $O(10)$ or $O(100)$, for instance. Then we need another suppression mechanism to bring down say, an $O(10)$ disorder to an $O(1)$ disorder at $M_{\mathrm{SUSY}}$. In this section we would like to discuss the second stage of suppressing FCNCs and CP phases. As shown in [18, 19, 20, 21], the IR attractive force of gauge interactions can be amplified in extra dimensions, driving the SSB terms to their infrared attractive values at the compactification scale $\Lambda_{C}$. If the disordered SSB terms at the cutoff scale $\Lambda$ converge rapidly to their IR fixed values that are flavor blind, FCNCs and dangerous CP violating phases can be desirably suppressed.

\section{A. A model}

Let us consider embedding the supersymmetric extension of the $S_{3}$ invariant SM of Sect. II into $\delta$ extra dimensions compactified on an orbifold with a compactification scale $\Lambda_{C}=$ 
$1 / R$. We assume that the matter multiplets and the $U(1)_{Y}$ gauge multiplet are located at a fixed point, while the $S U(2)_{L}$ and $S U(3)_{C}$ gauge multiplets propagate in the bulk. Therefore, only the KK tower of the $S U(2)_{L}$ and $S U(3)_{C}$ gauge multiplets contributes to the power running of parameters. Thanks to the dominance of the power running, we may ignore the logarithmic contributions. We, however, include those logarithmic corrections that come from $g_{1}$. Under this approximation we have computed the $\beta$ functions for the rigid and soft breaking parameters, which are given in Appendix.

First we recall that the KK tower of the $U(1)_{Y}$ gauge multiplet would not contribute to the $\beta$ function of $g_{1}$ and hence that of $M_{1}$ (at the one-loop level). So, they would be basically constant. However, they would contribute to the $\beta$ functions of the Yukawa couplings and hence to those of the SSB parameters. Since $g_{1}$ and $M_{1}$ remain approximately constant (while the other couplings become smaller) as $\Lambda$ increases, the contributions of the $U(1)_{Y}$ KK modes to the RG evolution would become dominant at high energies. As a consequence, we obtain a power dependence of $\Lambda$ instead of the logarithmic dependence (which is hidden in $\varepsilon_{2,3}$ of the solutions (59), (61) (62)). In other words, the uncertainty at high energy would remain unsuppressed at low energy, which would not be desirable.

Next let us discuss why we include the contributions from $g_{1}$. The discussion will also clarify the restriction of the suppression mechanism of the model. As we see from (A1) and (A4), the $U(1)_{Y}$ gauge coupling $g_{1}$ and the corresponding gaugino mass $M_{1}$ are running only logarithmically above $\Lambda_{C}$. So they are basically constant compared with other power-lawrunning parameters. To see this more in detail, we recall the analytic solutions for the RG evolution equations

$$
\begin{aligned}
& \frac{1}{g_{1}^{2}(\Lambda)}=\frac{1}{g_{1}^{2}\left(\Lambda_{C}\right)}-\frac{2\left(\sum l(R)\right)}{16 \pi^{2}} \log \frac{\Lambda}{\Lambda_{C}}, \\
& \frac{1}{g_{2}^{2}(\Lambda)}=\frac{1}{g_{2}^{2}\left(\Lambda_{C}\right)}+\frac{C_{2}(S U(2)) X_{\delta}}{8 \pi^{2} \delta}\left\{\left(\frac{\Lambda}{\Lambda_{C}}\right)^{\delta}-1\right\}, \\
& \frac{1}{g_{3}^{2}(\Lambda)}=\frac{1}{g_{3}^{2}\left(\Lambda_{C}\right)}+\frac{C_{2}(S U(3)) X_{\delta}}{8 \pi^{2} \delta}\left\{\left(\frac{\Lambda}{\Lambda_{C}}\right)^{\delta}-1\right\},
\end{aligned}
$$

where $\left(\sum l(R)\right)=39 / 5, C_{2}(S U(N))=N$. For $\Lambda / \Lambda_{C}>>1$, Eqs. (51) and (521) may be 
further approximated to

$$
\begin{aligned}
{\left[\frac{g_{a}\left(\Lambda_{C}\right)}{g_{a}(\Lambda)}\right]^{2} } & \simeq\left[\frac{C_{2}\left(G_{a}\right) X_{\delta} g_{a}^{2}\left(\Lambda_{C}\right)}{8 \pi^{2} \delta}\right]\left(\frac{\Lambda}{\Lambda_{C}}\right)^{\delta}(a=2,3) \\
& \simeq\left\{\begin{array}{c}
\left(0.02 \Lambda / \Lambda_{C}\right) \text { for } a=2 \\
\left(0.1 \Lambda / \Lambda_{C}\right) \text { for } a=3
\end{array} \text { with } \delta=1\right. \\
& \simeq\left\{\begin{array}{c}
\left(0.017 \Lambda / \Lambda_{C}\right)^{2} \text { for } a=2 \\
\left(0.09 \Lambda / \Lambda_{C}\right)^{2} \text { for } a=3
\end{array} \text { with } \delta=2,\right.
\end{aligned}
$$

where $X_{\delta}=\pi^{\delta / 2} / \Gamma(1+\delta / 2)=2(\pi)$ for $\delta=1(2)$ has been used, and we have assumed that $g_{2}^{2}\left(M_{Z}\right) \simeq g_{2}^{2}\left(\Lambda_{C}\right) \simeq 0.034 \times 4 \pi$ and $g_{3}^{2}\left(M_{Z}\right) \simeq g_{3}^{2}\left(\Lambda_{C}\right) \simeq 0.12 \times 4 \pi$. For the gaugino masses we obtain

$$
M_{a}\left(\Lambda_{C}\right)=\left(\frac{g_{a}\left(\Lambda_{C}\right)}{g_{a}(\Lambda)}\right)^{2} M_{a}(\Lambda), \quad a=1,2,3
$$

Note that $M_{1}$ does not get a power enhancement in contrast to $M_{2,3}$, because $\left(g_{1}\left(\Lambda_{C}\right) / g_{1}(\Lambda)\right)^{2}$ is only logarithmic. Therefore, $M_{1}$ should be much larger than $M_{2,3}$ at the cutoff scale $\Lambda$, if $M_{1}\left(\Lambda_{C}\right)$ should be $O\left(M_{2,3}\left(\Lambda_{C}\right)\right)$. The logarithmic running of $g_{1}$ and $M_{1}$ has an important consequence on the soft scalar mass matrix for the right-handed sleptons $m_{E_{R}}^{2}$. As we see from (A19), their $\beta$ functions depend only on $g_{1}$ and $M_{1}$, and one finds that

$$
\left(m_{E_{R}}^{2}\right)_{j}^{i}\left(\Lambda_{C}\right)=\left(m_{E_{R}}^{2}\right)_{j}^{i}(\Lambda)+\delta_{j}^{i} \frac{24}{5} \varepsilon_{3}\left|M_{1}\right|^{2}\left(\Lambda_{C}\right),
$$

where $i, j=1,2,3$ and

$$
\begin{aligned}
\varepsilon_{n} & =\frac{g_{1}^{2}\left(\Lambda_{C}\right)}{16 \pi^{2}} \int_{\Lambda_{C}}^{\Lambda} \frac{d \Lambda}{\Lambda}\left(\frac{g_{1}\left(\Lambda_{C}\right)}{g_{1}(\Lambda)}\right)^{2 n} \\
& =\frac{g_{1}^{2}\left(\Lambda_{C}\right)}{16 \pi^{2}} \ln \left(\Lambda / \Lambda_{C}\right)+O\left(g_{1}^{4}\right) .
\end{aligned}
$$

So, $m_{E_{R}}^{2}$, too, get only logarithmic corrections, which means that $m_{E_{R}}^{2}\left(\Lambda_{C}\right) \sim m_{E_{R}}^{2}(\Lambda)$. Therefore, $m_{E_{R}}^{2}(\Lambda)$ should be much larger than $M_{2,3}^{2}(\Lambda)$; too small $m_{E_{R}}^{2}\left(\Lambda_{C}\right)$ are phenomenologically not acceptable (in contrast to $M_{1}\left(\Lambda_{C}\right)$ ). However, if $m_{E_{R}}^{2}(\Lambda)>>M_{2}^{2}(\Lambda)$, say $m_{E_{R}}^{2}(\Lambda) \sim\left(10^{3} M_{2}(\Lambda)\right)^{2}$, this would be a unnatural fine tuning at the cutoff scale $\Lambda$, and would be against our philosophy of the present paper. So, we require

$$
\left|m_{E_{R}}^{2}\right|(\Lambda) \lesssim\left(10\left|M_{2}\right|(\Lambda)\right)^{2}
$$


for the fine tuning not to be unnatural. This is a strong restriction of the suppression mechanism for the present model, on one hand, because it limits $M_{2}\left(\Lambda_{C}\right) / M_{2}(\Lambda)$ and hence $\Lambda / \Lambda_{C}$ which is the power-law enhancement factor (53). On the other hand, it is a prediction of the model that $M_{1}\left(\Lambda_{C}\right)$ and $m_{E_{R}}\left(\Lambda_{C}\right)$ are smaller than the other gaugino masses and soft scalar masses. Further, as we see from (155), $\left(m_{E_{R}}^{2}\right)_{j}^{i}$ do not converge to IR attractive values. Fortunately, as we can see from Table I and (40), the experimental bound on $\delta_{R R}$ 's are very weak; it is sufficient to satisfy

$$
\Delta a_{R}^{\ell}<5 \times 10^{2}\left(\frac{m_{\ell}}{100 \mathrm{GeV}}\right)^{2}
$$

\section{B. Suppression of FCNCs}

Keeping the above discussions in mind, we proceed with our consideration of the IR attractiveness of the other SSB parameters. Using the $\beta$ functions given in Appendix, we find the following analytic solutions for the RG evolution equations.

\section{Quark sector:}

$$
\begin{aligned}
\frac{h_{k}^{D}}{Y_{k}^{D}}\left(\Lambda_{C}\right) & =\frac{h_{k}^{D}}{Y_{k}^{D}}(\Lambda)-\frac{14}{15} \varepsilon_{2} M_{1}\left(\Lambda_{C}\right)-\frac{12}{8}(1-c) M_{2}\left(\Lambda_{C}\right)-\frac{16}{9}(1-d) M_{3}\left(\Lambda_{C}\right) \\
\frac{h_{k}^{U}}{Y_{k}^{U}}\left(\Lambda_{C}\right) & =\frac{h_{k}^{U}}{Y_{k}^{U}}(\Lambda)-\frac{26}{15} \varepsilon_{2} M_{1}\left(\Lambda_{C}\right)-\frac{12}{8}(1-c) M_{2}\left(\Lambda_{C}\right)-\frac{16}{9}(1-d) M_{3}\left(\Lambda_{C}\right) \\
\left(m_{Q}^{2}\right)_{j}^{i}\left(\Lambda_{C}\right) & =\left(m_{Q}^{2}\right)_{j}^{i}(\Lambda)+\delta_{j}^{i}\left[\frac{2}{15} \varepsilon_{3}\left|M_{1}\right|^{2}\left(\Lambda_{C}\right)+\frac{6}{8}\left(1-c^{2}\right)\left|M_{2}\right|^{2}\left(\Lambda_{C}\right)+\frac{32}{36}\left(1-d^{2}\right)\left|M_{3}\right|^{2}\left(\Lambda_{C}\right)\right], \\
\left(m_{U_{R}}^{2}\right)_{j}^{i}\left(\Lambda_{C}\right) & =\left(m_{U_{R}}^{2}\right)_{j}^{i}(\Lambda)+\delta_{j}^{i}\left[\frac{32}{15} \varepsilon_{3}\left|M_{1}\right|^{2}\left(\Lambda_{C}\right)+\frac{32}{36}\left(1-d^{2}\right)\left|M_{3}\right|^{2}\left(\Lambda_{C}\right)\right] \\
\left(m_{D_{R}}^{2}\right)_{j}^{i}\left(\Lambda_{C}\right) & =\left(m_{D_{R}}^{2}\right)_{j}^{i}(\Lambda)+\delta_{j}^{i}\left[\frac{8}{15} \varepsilon_{3}\left|M_{1}\right|^{2}\left(\Lambda_{C}\right)+\frac{32}{36}\left(1-d^{2}\right)\left|M_{3}\right|^{2}\left(\Lambda_{C}\right)\right]
\end{aligned}
$$

where $k=1,2,3,4,5, i, j=1,2,3, \varepsilon_{n}$ is defined in (56), and

$$
c=\left(\frac{g_{2}(\Lambda)}{g_{2}\left(\Lambda_{C}\right)}\right)^{2}, d=\left(\frac{g_{3}(\Lambda)}{g_{3}\left(\Lambda_{C}\right)}\right)^{2} .
$$

\section{Lepton sector:}




$$
\begin{aligned}
\frac{h_{k}^{E}}{Y_{k}^{E}}\left(\Lambda_{C}\right) & =\frac{h_{k}^{E}}{Y_{k}^{E}}(\Lambda)-\frac{18}{5} \varepsilon_{2} M_{1}\left(\Lambda_{C}\right)-\frac{12}{8}(1-c) M_{2}\left(\Lambda_{C}\right), \\
\frac{h_{k}^{N}}{Y_{k}^{N}}\left(\Lambda_{C}\right) & =\frac{h_{k}^{N}}{Y_{k}^{N}}(\Lambda)-\frac{6}{5} \varepsilon_{2} M_{1}\left(\Lambda_{C}\right)-\frac{12}{8}(1-c) M_{2}\left(\Lambda_{C}\right), \\
\left(m_{L}^{2}\right)_{j}^{i}\left(\Lambda_{C}\right) & =\left(m_{L}^{2}\right)_{j}^{i}(\Lambda)+\delta_{j}^{i}\left[\frac{6}{5} \varepsilon_{3}\left|M_{1}\right|^{2}\left(\Lambda_{C}\right)+\frac{6}{8}\left(1-c^{2}\right)\left|M_{2}\right|^{2}\left(\Lambda_{C}\right)\right], \\
\left(m_{E_{R}}^{2}\right)_{j}^{i}\left(\Lambda_{C}\right) & =\left(m_{E_{R}}^{2}\right)_{j}^{i}(\Lambda)+\delta_{j}^{i} \frac{24}{5} \varepsilon_{3}\left|M_{1}\right|^{2}\left(\Lambda_{C}\right),
\end{aligned}
$$

where $k=2,4,5(2,3,4)$ for the charged lepton(neutrino) sector and $i, j=1,2,3$. The Majorana neutrino masses, $M_{1,3}$, and their soft scalar masses, $\left(m_{N_{R}}^{2}\right)_{j}^{i}$, have only a logarithmic running.

\section{Higgs sector:}

$$
\begin{aligned}
& \frac{B_{k}}{\mu_{k}}\left(\Lambda_{C}\right)=\frac{B_{k}}{\mu_{k}}(\Lambda)-\frac{6}{5} \varepsilon_{2} M_{1}\left(\Lambda_{C}\right)-\frac{12}{8}(1-c) M_{2}\left(\Lambda_{C}\right), \quad k=1,3, \\
& m_{a}^{2}\left(\Lambda_{C}\right)=m_{a}^{2}(\Lambda)+\frac{6}{5} \varepsilon_{3}\left|M_{1}\right|^{2}\left(\Lambda_{C}\right)+\frac{6}{8}\left(1-c^{2}\right)\left|M_{2}\right|^{2}\left(\Lambda_{C}\right), \quad a=H^{U}, H^{D} .
\end{aligned}
$$

As it is clear, the quantities on the right-hand side of (59), (61) and (62) whose argument is $\Lambda$ are initial values at $\Lambda$, and those of $\Lambda_{C}$ are IR attractive values. Therefore, $\Delta a$ 's and also $\tilde{A}$ 's, given in (39), at $\Lambda_{C}$ can be written in terms of the initial values. We emphasize that all the infrared attractive values are flavor diagonal.

To estimate the suppression of the initial values at $\Lambda$, we assume that the initial SSB parameters are disordered around $M_{2}(\Lambda)$. For instance,

$$
A_{1}^{U}(\Lambda)=\frac{h_{1}^{U}}{Y_{1}^{U}}(\Lambda)=\kappa_{1}^{U} M_{2}(\Lambda), m_{Q}^{2}=\kappa_{m_{Q}}^{2} M_{2}^{2}(\Lambda),
$$

and similarly for other SSB parameters, where $\kappa$ 's are unknown parameters. Then we obtain

$$
\Delta \tilde{A}_{i j}^{q}\left(\Lambda_{C}\right)=\frac{A_{i}^{q}-A_{j}^{q}}{m_{\tilde{q}}}\left(\Lambda_{C}\right)=\Delta \kappa_{i j}^{q} \frac{M_{2}(\Lambda)}{m_{\tilde{q}}\left(\Lambda_{C}\right)}=\Delta \kappa_{i j}^{q} \frac{M_{2}\left(\Lambda_{C}\right)}{m_{\tilde{q}}\left(\Lambda_{C}\right)} \frac{M_{2}(\Lambda)}{M_{2}\left(\Lambda_{C}\right)},
$$

where $\Delta \kappa_{i j}^{q}=\kappa_{i}^{q}-\kappa_{j}^{q}$ with $i, j=1, \ldots, 5$. Similarly, we find

$$
\Delta \tilde{A}_{i j}^{\ell}\left(\Lambda_{C}\right)=\Delta \kappa_{i j}^{\ell} \frac{M_{2}\left(\Lambda_{C}\right)}{m_{\tilde{\ell}}\left(\Lambda_{C}\right)} \frac{M_{2}(\Lambda)}{M_{2}\left(\Lambda_{C}\right)}, i, j=2,4,5
$$




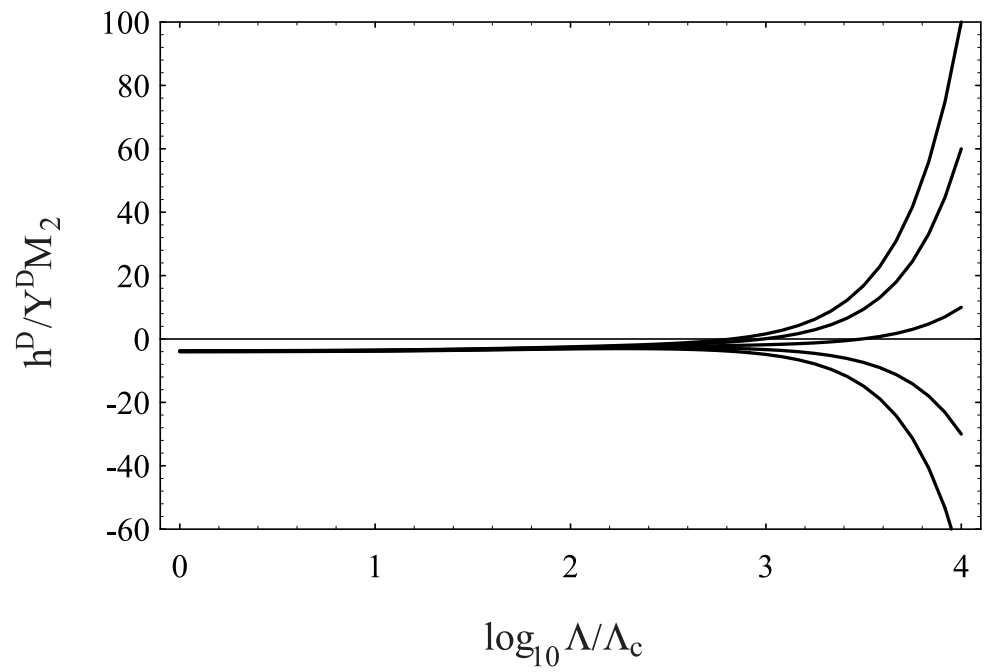

FIG. 1: The IR attractiveness of $h_{D} /\left(M_{2} Y_{D}\right)$.

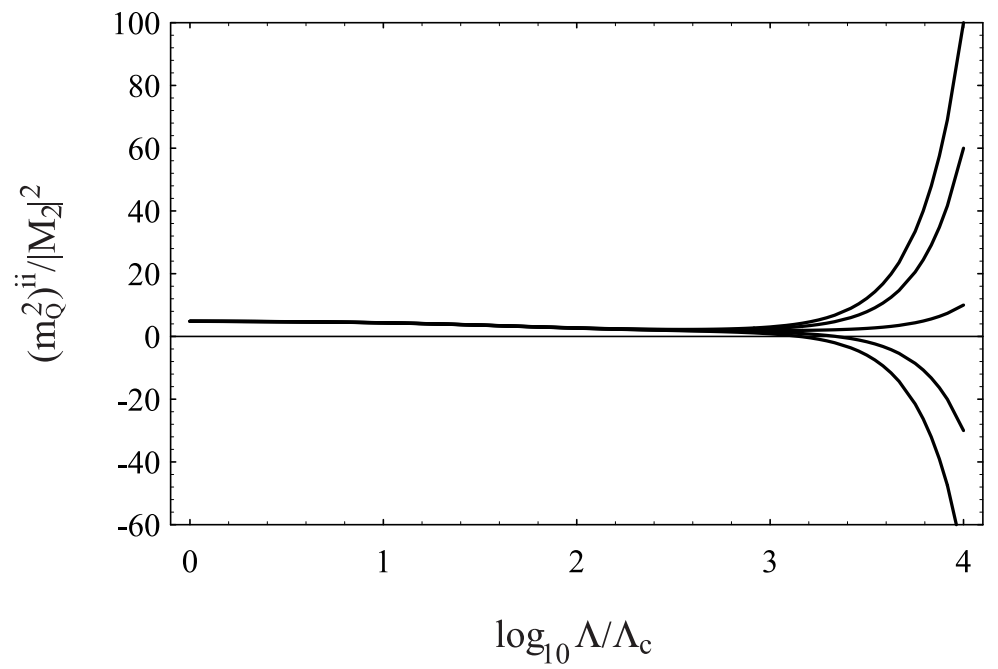

FIG. 2: The IR attractiveness of $m_{Q}^{2} /\left|M_{2}\right|^{2}$.

and

$$
\Delta a^{q}=\Delta \kappa_{m_{q}}^{2}\left[\frac{M_{2}\left(\Lambda_{C}\right)}{m_{\tilde{q}}\left(\Lambda_{C}\right)}\right]^{2}\left[\frac{M_{2}(\Lambda)}{M_{2}\left(\Lambda_{C}\right)}\right]^{2}, \Delta a^{\ell}=\Delta \kappa_{m_{\ell}}^{2}\left[\frac{M_{2}\left(\Lambda_{C}\right)}{m_{\tilde{\ell}}\left(\Lambda_{C}\right)}\right]^{2}\left[\frac{M_{2}(\Lambda)}{M_{2}\left(\Lambda_{C}\right)}\right]^{2} .
$$

In (66) we have suppressed the prefixes $L$ and $R$, because they have the same form. To be more explicit, we assume

$$
m_{E_{R}}\left(\Lambda_{C}\right) \gtrsim 100 \mathrm{GeV} \text { and } M_{2}\left(\Lambda_{C}\right) \lesssim 1 \mathrm{TeV},
$$

so that Eq. (57) implies that $M_{2}(\Lambda) \gtrsim 10 \mathrm{GeV}$. This means

$$
\frac{M_{2}(\Lambda)}{M_{2}\left(\Lambda_{C}\right)}>10^{-2}
$$


With this value of the enhancement factor, we obtain

$$
\Delta \tilde{A}\left(\Lambda_{C}\right) \gtrsim 10^{-2} \Delta \kappa \text { and } \Delta a\left(\Lambda_{C}\right) \gtrsim 10^{-4} \Delta \kappa^{2}
$$

where we have assumed that $m_{\tilde{\ell}} \simeq m_{\tilde{q}} \simeq M_{2}$ at $\Lambda_{C}$. In Figs. 1 and 2 we show the IR attractiveness of $h_{D} / M_{2} Y_{D}$ and $m_{Q}^{2} /\left|M_{2}\right|^{2}$ for $\delta=1$. The constraint (68) with (53) implies that $\Lambda / \Lambda_{C} \lesssim 5 \times 10^{3}$ in this case. Since between $\Lambda_{C}$ and $M_{\text {SUSY }}$ there are only logarithmic corrections, we may assume that the inequalities (69) remain unchanged at $M_{\mathrm{SUSY}}$. As we have seen in the previous section, $\Delta \tilde{A} \lesssim 1$ and $\Delta a \lesssim 1$ are sufficient (except for the fine tuning (49) to suppress the contributions to EDM of the neutron) to suppress FCNCs and dangerous $\mathrm{CP}$ violating phases at $M_{\mathrm{SUSY}}$. Therefore, at most an $O(100)$ disorder of the SSB parameters at $\Lambda$ may be allowed.

Once again, $S_{3}$ symmetry can suppress sufficiently FCNCs and CP phases that are generated by the SSB parameters in $O(1)$ disorder at $M_{\mathrm{SUSY}}$, and the asymptotically free $S U(2)_{L}$ and $S U(3)_{C}$ gauge interactions in extra dimensions can bring the SSB parameters in $O(100)$ disorder at the cutoff scale down to those in $O(1)$ disorder at the compactification scale. In this way, FCNCs and CP phases enjoy a double suppression.

\section{CONCLUSIONS}

A low energy nonabelian flavor symmetry is certainly a powerful tool to suppress flavorchanging neutral currents (FCNCs) and CP violating phases that are induced by soft supersymmetry breaking (SSB) terms. In the case of the MSSM, where only two types of Higgs supermultiplets $H^{U}$ and $H^{D}$ exist, any nonabelian flavor symmetry has to be explicitly broken to describe experimental data. Therefore, it would be unnatural to assume a flavor symmetry only in the SSB sector. However, if the Higgs sector is extended, and the Higgs supermultiplets belong to a non-trivial representation of a flavor group, phenomenologically viable possibilities arise. We have considered the smallest nonabelian group $S_{3}$ as a flavor group to extend the MSSM. Under this flavor group, not only the fermion multiplets, but also the two types of the Higgs multiplets belong to the three dimensional representation of $S_{3}$ [34, 35]. The most general Higgs superpotential has an accidental continuous global symmetry, implying that a lot of pseudo Goldstone bosons will appear after spontaneous symmetry breaking of the electroweak symmetry [31]. To overcome this problem, we intro- 
duced soft $S_{3}$ breaking B terms in the SSB sector, because violation of $S_{3}$ can be confined in this sector. We found that in this model FCNCs and CP phases, which are induced by the SSB parameters in $O(1)$ disorder at $M_{\text {SUSY }}$, are sufficiently suppressed to satisfy the experimental constraints.

Then we extended the $S_{3}$ invariant supersymmetric model into $\delta$ extra dimensions compactified on an orbifold with a compactification scale $\Lambda_{C}=1 / R$, and assumed that the matter multiplets and the $U(1)_{Y}$ gauge multiplet are located at a fixed point, while the $S U(2)_{L}$ and $S U(3)_{C}$ gauge multiplets propagate in the bulk. In this way, the $S U(2)_{L}$ and $S U(3)_{C}$ gauge couplings become asymptotically free, while the running of the $U(1)_{Y}$ coupling is frozen. These asymptotically free gauge couplings amplify the infrared attractiveness of the SSB parameters when running from the cutoff scale down to the compactification scale [18, 19, 20, 21]. We found (69) that thanks to the double suppression mechanism, a disorder of two orders of magnitude in the SSB parameters at the cutoff scale may be allowed to satisfy experimental constraints on FCNC processes and $\mathrm{CP}$ violating phenomena.

\section{Acknowledgments}

This work(Y.K. and J.K.) is supported by the Grants-in-Aid for Scientific Research from the Japan Society for the Promotion of Science (No. 13135210). K.Y.C. is supported in part by the KOSEF ABRL Grant to Particle Theory Research Group of SNU, the BK21 program of Ministry of Education, and Korea Research Foundation Grant No. KRF-PBRG2002-070-C00022. This work(H.M.L.) is supported by the European Community's Human Potential Programme under contracts HPRN-CT-2000-00131 Quantum Spacetime, HPRNCT-2000-00148 Physics Across the Present Energy Frontier and HPRN-CT-2000-00152 Supersymmetry and the Early Universe. H.M.L. was supported by priority grant 1096 of the Deutsche Forschungsgemeinschaft.

\section{APPENDIX A: RENORMALIZATION GROUP EQUATIONS}

Here we give the $\beta$ functions. The model in Sect. IV is a simple embedding of the supersymmetric extension of the $S_{3}$ invariant SM into extra dimensions compactified on an orbifold [23, 25, 26], where it is assumed that the matter multiplets and the $U(1)_{Y}$ 
gauge multiplet are located at a fixed point, while the $S U(2)_{L}$ and $S U(3)_{c}$ gauge multiplets propagate in the bulk. The dominance of the power running yields the approximate $\beta$ functions that are given below ${ }^{5}$.

\section{Gauge sector:}

$$
\begin{aligned}
16 \pi^{2} \beta_{g_{1}} & =\frac{1}{16 \pi^{2}}\left(\sum l(R)\right) g_{1}^{3} \\
16 \pi^{2} \beta_{g_{2}} & =-\frac{1}{16 \pi^{2}} C_{2}(S U(2)) F_{\delta}^{2} g_{2}^{3}, \\
16 \pi^{2} \beta_{g_{3}} & =-\frac{1}{16 \pi^{2}} C_{2}(S U(3)) F_{\delta}^{2} g_{3}^{3}, \\
16 \pi^{2} \beta_{M_{1}} & =\frac{2}{16 \pi^{2}}\left(\sum l(R)\right) g_{1}^{2} M_{1} \\
16 \pi^{2} \beta_{M_{2}} & =-\frac{2}{16 \pi^{2}} C_{2}(S U(2)) F_{\delta}^{2} g_{2}^{2} M_{2}, \\
16 \pi^{2} \beta_{M_{3}} & =-\frac{2}{16 \pi^{2}} C_{2}(S U(3)) F_{\delta}^{2} g_{3}^{2} M_{3},
\end{aligned}
$$

where $F_{\delta}=X_{\delta}^{1 / 2}(R \Lambda)^{\delta / 2}$ with $X_{\delta}=\pi^{\delta / 2} / \Gamma(1+\delta / 2)=2(\pi)$ for $\delta=1(2)$, the quadratic Casimir is given by $C_{2}(S U(N))=N$, and $\sum l(R)=39 / 5$. As explained in section III, we ignore logarithmic contributions except those from $g_{1}$.

\section{Quark sector:}

\footnotetext{
${ }^{5}$ We note that, even with the reduction of the number of the KK modes on the orbifold, the coupling of the KK modes to the brane fields is larger than the one of the zero mode by $\sqrt{2}$ due to the difference of normalization of mode functions.
} 


$$
\begin{aligned}
16 \pi^{2} \beta_{Y_{k}^{D}} & =Y_{k}^{D}\left(-\frac{7}{15} g_{1}^{2}-3 g_{2}^{2} F_{\delta}^{2}-\frac{16}{3} g_{3}^{2} F_{\delta}^{2}\right), \\
16 \pi^{2} \beta_{Y_{k}^{U}} & =Y_{k}^{U}\left(-\frac{13}{15} g_{1}^{2}-3 g_{2}^{2} F_{\delta}^{2}-\frac{16}{3} g_{3}^{2} F_{\delta}^{2}\right), \\
16 \pi^{2} \beta_{h_{k}^{D}} & =\frac{7}{15} g_{1}^{2}\left(2 M_{1} Y_{k}^{D}-h_{k}^{D}\right)+3 g_{2}^{2} F_{\delta}^{2}\left(2 M_{2} Y_{k}^{D}-h_{k}^{D}\right) \\
& +\frac{16}{3} g_{3}^{2} F_{\delta}^{2}\left(2 M_{3} Y_{k}^{D}-h_{k}^{D}\right), \\
16 \pi^{2} \beta_{h_{k}^{U}} & =\frac{13}{15} g_{1}^{2}\left(2 M_{1} Y_{k}^{U}-h_{k}^{U}\right)+3 g_{2}^{2} F_{\delta}^{2}\left(2 M_{2} Y_{k}^{U}-h_{k}^{U}\right) \\
& +\frac{16}{3} g_{3}^{2} F_{\delta}^{2}\left(2 M_{3} Y_{k}^{U}-h_{k}^{U}\right),
\end{aligned}
$$

where $k=1,2,3,4,5$.

$$
\begin{aligned}
16 \pi^{2}\left(\beta_{m_{Q}^{2}}\right)_{j}^{i} & =\delta_{j}^{i}\left[-\frac{2}{15}\left|M_{1}\right|^{2} g_{1}^{2}-6\left|M_{2}\right|^{2} g_{2}^{2} F_{\delta}^{2}-\frac{32}{3}\left|M_{3}\right|^{2} g_{3}^{2} F_{\delta}^{2}\right], \\
16 \pi^{2}\left(\beta_{m_{U_{R}}^{2}}\right)_{j}^{i} & =\delta_{j}^{i}\left[-\frac{32}{15}\left|M_{1}\right|^{2} g_{1}^{2}-\frac{32}{3}\left|M_{3}\right|^{2} g_{3}^{2} F_{\delta}^{2}\right], \\
16 \pi^{2}\left(\beta_{m_{D_{R}}^{2}}\right)_{j}^{i} & =\delta_{j}^{i}\left[-\frac{8}{15}\left|M_{1}\right|^{2} g_{1}^{2}-\frac{32}{3}\left|M_{3}\right|^{2} g_{3}^{2} F_{\delta}^{2}\right]
\end{aligned}
$$

where $i, j=1,2,3$.

\section{Lepton sector:}

$$
\begin{gathered}
16 \pi^{2} \beta_{Y_{k}^{E}}=Y_{k}^{E}\left(-\frac{9}{5} g_{1}^{2}-3 g_{2}^{2} F_{\delta}^{2}\right), \quad k=2,4,5, \\
16 \pi^{2} \beta_{Y_{k}^{N}}=Y_{k}^{N}\left(-\frac{3}{5} g_{1}^{2}-3 g_{2}^{2} F_{\delta}^{2}\right), \quad k=2,3,4 . \\
16 \pi^{2} \beta_{h_{k}^{E}}=\frac{9}{5} g_{1}^{2}\left(2 M_{1} Y_{k}^{E}-h_{k}^{E}\right)+3 g_{2}^{2} F_{\delta}^{2}\left(2 M_{2} Y_{k}^{E}-h_{k}^{E}\right), \quad k=2,4,5, \\
16 \pi^{2} \beta_{h_{k}^{N}}=\frac{3}{5} g_{1}^{2}\left(2 M_{1} Y_{k}^{N}-h_{k}^{N}\right)+3 g_{2}^{2} F_{\delta}^{2}\left(2 M_{2} Y_{k}^{N}-h_{k}^{N}\right), k=2,3,4 . \\
16 \pi^{2}\left(\beta_{m_{L}^{2}}\right)_{j}^{i}=\delta_{j}^{i}\left[-\frac{6}{5}\left|M_{1}\right|^{2} g_{1}^{2}-6\left|M_{2}\right|^{2} g_{2}^{2} F_{\delta}^{2}\right] \\
16 \pi^{2}\left(\beta_{m_{E_{R}}^{2}}\right)_{j}^{i}=\delta_{j}^{i}\left[-\frac{24}{5}\left|M_{1}\right|^{2} g_{1}^{2}\right]
\end{gathered}
$$


where $i, j=1,2,3$.

$$
\begin{aligned}
16 \pi^{2}\left(\beta_{m_{N_{R}}^{2}}\right)_{J}^{I} & =\delta_{J}^{I}\left(4 X_{N_{2}}+2 X_{N_{4}}\right), \\
16 \pi^{2}\left(\beta_{m_{N_{R}}^{2}}\right)_{3}^{3} & =2 X_{N_{3}}, \\
16 \pi^{2} \beta_{M_{1}} & =2 M_{1}\left(4\left|Y_{2}^{N}\right|^{2}+2\left|Y_{4}^{N}\right|^{2}\right), \\
16 \pi^{2} \beta_{M_{3}} & =2 M_{3}\left(2\left|Y_{3}^{N}\right|^{2}\right)
\end{aligned}
$$

where $I, J=1,2$ and sneutrino masses have no power running.

\section{Higgs sector:}

$$
\begin{aligned}
& 16 \pi^{2} \beta_{\mu_{k}}= \mu_{k}\left[-\frac{3}{5} g_{1}^{2}-3 g_{2}^{2} F_{\delta}^{2}\right], \quad k=1,3, \\
& 16 \pi^{2} \beta_{B_{k}}=\left\{\begin{array}{l}
\frac{3}{5} g_{1}^{2}\left(2 M_{1} \mu_{k}-B_{k}\right)+3 g_{2}^{2} F_{\delta}^{2}\left(2 M_{2} \mu_{k}-B_{k}\right), \quad k=1,3, \\
\frac{3}{5} g_{1}^{2}\left(-B_{k}\right)+3 g_{2}^{2} F_{\delta}^{2}\left(-B_{k}\right), \quad k=4,5,6,7 .
\end{array}\right. \\
& 16 \pi^{2}\left(\beta_{m_{a}^{2}}\right)_{j}^{i}=\delta_{j}^{i}\left[-\frac{6}{5}\left|M_{1}\right|^{2} g_{1}^{2}-6\left|M_{2}\right|^{2} g_{2}^{2} F_{\delta}^{2}\right]
\end{aligned}
$$

where $a=H^{U}, H^{D}$ and $i, j=1,2,3$.

[1] For a recent review, see, for instance, G. L. Kane, hep-ph/0202185, and references therein.

[2] S. Dimopoulos and D. Sutter, Nucl. Phys. B452, 496 (1995).

[3] L. Hall, V.A. Kostelecky and S. Raby, Nucl. Phys. B267, 415 (1986); F. Gabbiani and A. Masiero, Phys. Lett. B209, 289 (1988).

[4] J. Ellis and D.V. Nanopoulos, Phys. Lett. B110, 44 (1982); R. Barbieri and R. Gatto, Phys. Lett. B110, 211 (1982); B. Campbell, Phys. Rev. D 28, 209 (1983); M.J. Duncan, Nucl. Phys. B221, 285 (1983); J.F. Donoghue and H.P. Nilles and D. Wyler, Phys. Lett. B128, 55 (1983); J.-M. Gérard, W. Grimus, A. Raychaudhuri and G. Zoupanos, Phys. Lett. B140, 349 (1984).

[5] J. Ellis, S. Ferrara and D.V. Nanopoulos, Phys. Lett. B114, 231 (1982); W. Buchmüller and D. Wyler, Phys. Lett. B121, 321 (1983); J. Polchinski and M.B. Wise, Phys. Lett. B125, 
393 (1983); F. del Aguila, M.B. Gavela, J.A. Grifols and A. Méndez, Phys. Lett. B126, 71 (1983); D.V. Nanopoulos and M. Srednicki, Phys. Lett. B128, 61 (1983).

[6] S. Bertolini, F. Borzumati, A. Masiero and G. Ridolfi, Nucl. Phys. B353, 591 (1991); R. Barbieri and G.F. Giudice, Phys. Lett. B309, 86 (1993).

[7] F. Gabbiani, E. Gabrielli, A. Masiero and L. Silvestrini, Nucl. Phys. B477, 321 (1996).

[8] S. Dimopoulos and H. Georgi, Nucl. Phys. B193, 150 (1981); N. Sakai, Z. Phys. C11, 153 (1981).

[9] M. Dine and A. E. Nelson, Phys. Rev. D48, 1277 (1993); M. Dine, A. E. Nelson and Y. Shirman, Phys. Rev. D51, 1362 (1995); For a review, G. F. Giudice and R. Rattazzi, Phys. Rept. 322, 419 (1999).

[10] L. Randall and R. Sundrum, Nucl. Phys. B557, 79 (1999); G. F. Giudice, M. A. Luty, H. Murayama and R. Rattazi, JHEP 12, 027 (1998).

[11] D. E. Kaplan, G. D. Kribs and M. Schmaltz, Phys. Rev. D62, 035010 (2000); Z. Chacko, M. A. Luty, A. E. Nelson and E. Ponton, JHEP 01, 003 (2000).

[12] M. Lanzagorta and G.G. Ross, Phys. Lett. B364, 163 (1995); P.M. Ferreira, I. Jack and D.R.T. Jones, Phys. Lett. B357, 359 (1995); S.A. Abel and B.C. Allanach, Phys. Lett. B415, 371 (1997); S.F. King and G.G. Ross, Nucl. Phys. B530, 3 (1998); I. Jack and D.R.T. Jones, Phys. Lett. B443, 177 (1998); G.K. Yeghiyan, M. Jurcisin and D.I. Kazakov, Mod. Phys. Lett. A14, 601 (1999); M. Jurcisin and D.I. Kazakov, Mod. Phys. Lett. A14, 671 (1999).

[13] A. Karch, T. Kobayashi, J. Kubo, and G. Zoupanos, Phys. Lett. B441, 235 (1998); M. Luty and R. Rattazzi, JHEP 11, 001 (1999).

[14] A. E. Nelson and M. J. Strassler, JHEP 09, (2000) 030; T. Kobayashi and H. Terao, Phys. Rev. D64, 075003 (2001).

[15] T. Kobayashi , H. Nakano and H. Terao, Phys. Rev. D65, 015006 (2002); T. Kobayashi, H. Nakano, T. Noguchi and H. Terao, Phys. Rev. D66 095011 (2002); JHEP 0302, 022 (2003).

[16] M. A. Luty and R. Sundrum, Phys. Rev. D65, 066004 (2002); Phys. Rev. D67, 045007 (2003).

[17] S.A. Abel and S.F. King, Phys. Rev. D59, 095010 (1999); T. Kobayashi and K. Yoshioka, Phys. Rev. D62, 115003 (2000); M. Bando, T. Kobayashi, T. Noguchi and K. Yoshioka, Phys. Rev. D63, 113017 (2001).

[18] J. Kubo and H. Terao, Phys. Rev. D66, 116003 (2002). 
[19] K-S. Choi, K-Y. Choi and J.E. Kim, Phys. Rev. D68, 035003 (2003).

[20] K-Y. Choi, J.E. Kim and H. M. Lee, JHEP 0306, 040 (2003).

[21] Y. Kajiyama, J. Kubo and H. Terao, hep-ph/0311316.

[22] T. R. Taylor and G. Veneziano, Phys. Lett. B212, 147 (1988).

[23] K. Dienes, E. Dudas and T. Gherghetta, Nucl. Phys. B537, 47 (1999).

[24] S. Ejiri, J. Kubo and M. Murata, Phys. Rev. D62, 105025 (2000).

[25] I. Antoniadis, Phys. Lett. B246, 377 (1990); I. Antoniadis, C. Muñoz and M. Quirós, Nucl. Phys. B397, 515 (1993).

[26] N. Arkani-Hamed, S. Dimopoulos and G. Dvali, Phys. Rev. D59, 086004 (1999).

[27] T. Kobayashi, J. Kubo, M. Mondragon and G. Zoupanos, Nucl. Phys. B550, 99 (1999); J. Kubo, H. Terao and G. Zoupanos, Nucl. Phys. B574, 495 (2000).

[28] L.J. Hall and H. Murayama, Phys. Rev. Lett. 75 (1995) 3985; C.D. Carone, L.J. Hall and H. Murayama, Phys. Rev. D53, 6282 (1996).

[29] K. Hamaguchi, M. Kakizaki and M. Yamaguchi, Phys. Rev. D68, 056007 (2003).

[30] K.S. Babu, T. Kobayashi and J. Kubo, Phys. Rev. D67, 075018 (2003).

[31] T. Kobayashi, J. Kubo and H. Terao, Phys. Lett. B568, 83 (2003).

[32] S. F. King, L. Velasco-Sevilla and G. G. Ross, hep-ph/0401064.

[33] S. F. King and G. G. Ross, Phys. Lett. B520, 243 (2001); hep-ph/0307190 G. G. Ross and L. Velasco-Sevilla, Nucl.Phys. B653, 3 (2003).

[34] J. Kubo, A. Mondragón, M. Mondragón and E. Rodríguez-Jáuregui, Prog. Theor. Phys. 109, 795 (2003).

[35] J. Kubo, Phys. Lett. B578, 156 (2004).

[36] S. Pakvasa and H. Sugawara, Phys. Lett. B73, 61 (1978) ; Phys. Lett. B82,105 (1979); Y. Yamanaka, S. Pakvasa and H. Sugawara, Phys. Rev. D25, 1895 (1982); Erratum D29, 2135 (1984).

[37] H. Harari, H. Haut and T. Weyers, Phys. Lett. B78, 459 (1978).

[38] Y. Koide, Lett. Nuovo Cimento 34, 201 (1982) ; Phys. Lett. B120, 161 (1983); Phys. Rev. D28, 252 (1983).

[39] H. Fritzsch and Z.Z. Xing, Prog. Part. Nucl. Phys. 45, 1 (2000).

[40] E. Ma and G. Rajasekaran, Phys. Rev. D64, 113012 (2001); E. Ma, Mod. Phys. Lett. A17, 627 (2002); 2361 (2002). 
[41] K.S. Babu, E. Ma and J.W.F. Valle, Phys. Lett. B552, 207 (2003).

[42] M. Hirsch, J.C. Romao, S. Skadhauge, J.W.F. Valle and A. Villanova del Moral, hep-ph/0312265.

[43] W. Grimus and L. Lavoura, Phys. Lett. B572, 76 (2003).

[44] W. Grimus and L. Lavoura, JHEP 0107, 045 (2001); Acta Phys. Polon. B32, 3719 (2001).

[45] Y. Koide, Phys. Rev. D60, 077301 (1999); Y. Koide, H. Nishiura, K. Matsuda, T. Kikuchi and T. Fukuyama, Phys. Rev. D66, 093006 (2002).

[46] T. Ohlsson and G. Seidl, Phys. Lett. B537, 95 (2002); Nucl. Phys. B643, 247 (2002).

[47] T. Kitabayashi and M. Yasue, Phys .Rev. D67, 015006 (2003).

[48] E. Ma and G. Rajasekaran, Phys. Rev. D68, 071302 (2003 ); E. Ma, hep-ph/0308282 hep-ph/0401025.

[49] W. Grimus and L. Lavoura, hep-ph/0305309, hep-ph/0312330.

[50] A.Yu. Smirnov; hep-ph/0311259. 